High perceived stress is associated with unfavorable eating behavior in overweight and obese Finns of working age

\title{
Jarvela-Reijonen, Elina
}

2016-08-01

Jarvela-Reijonen, E , Karhunen , L, Sairanen , E, Rantala , S , Laitinen , J , Puttonen , S , Peuhkuri , K, Hallikainen, M , Juvonen , K, Myllymaki , T, Fohr , T , Pihlajamaki , J , Korpela , R , Ermes , M , Lappalainen , R \& Kolehmainen , M 2016 , ' High perceived stress is associated with unfavorable eating behavior in overweight and obese Finns of working age ' , Appetite , vol. 105 , pp. 249-258 . https://doi.org/10.1016/j.appet.2016.04.023

http://hdl.handle.net/10138/224090

https://doi.org/10.1016/j.appet.2016.04.023

publishedVersion

Downloaded from Helda, University of Helsinki institutional repository.

This is an electronic reprint of the original article.

This reprint may differ from the original in pagination and typographic detail.

Please cite the original version. 


\title{
High perceived stress is associated with unfavorable eating behavior in overweight and obese Finns of working age
}

\author{
Elina Järvelä-Reijonen ${ }^{\text {a, * }}$, Leila Karhunen ${ }^{\text {a, b }}$, Essi Sairanen ${ }^{\text {c }}$, Sanni Rantala ${ }^{\text {d, }}$ \\ Jaana Laitinen ${ }^{\text {, }}$ Sampsa Puttonen ${ }^{\text {, }}$ Katri Peuhkuri ${ }^{\mathrm{d}}$, Maarit Hallikainen ${ }^{\text {a }}$, \\ Kristiina Juvonen ${ }^{\text {a }}$, Tero Myllymäki ${ }^{c}$, Tiina Föhr ${ }^{\mathrm{f}}$, Jussi Pihlajamäki ${ }^{\mathrm{a}, \mathrm{b}}$, Riitta Korpela ${ }^{\mathrm{d}}$, \\ Miikka Ermes ${ }^{g}$, Raimo Lappalainen ${ }^{\mathrm{c}}$, Marjukka Kolehmainen a, b \\ a Institute of Public Health and Clinical Nutrition, Clinical Nutrition, University of Eastern Finland, P.O. Box 1627, FI-70211 Kuopio, Finland \\ ${ }^{\mathrm{b}}$ Institute of Clinical Medicine and Clinical Nutrition, Kuopio University Hospital, P.O. Box 100, FI-70029 KYS, Finland \\ c Department of Psychology, University of Jyväskylä, P.O. Box 35, FI-40014, University of Jyväskylä, Finland \\ ${ }^{\mathrm{d}}$ Medical Faculty, Pharmacology, Medical Nutrition Physiology, University of Helsinki, P.O. Box 63, FI-00014, University of Helsinki, Helsinki, Finland \\ e Finnish Institute of Occupational Health, P.O. Box 40, FI-00251 Helsinki, Finland \\ ${ }^{\mathrm{f}}$ Department of Health Sciences, University of Jyväskylä, P.O. Box 35, FI-40014 Jyväskylä, Finland \\ ${ }^{g}$ VTT Technical Research Centre of Finland, P.O. Box 1300, FI-33101 Tampere, Finland
}

\section{A R T I C L E I N F O}

\section{Article history:}

Received 23 December 2015

Received in revised form

2 April 2016

Accepted 19 April 2016

Available online 21 April 2016

\section{Keywords:}

Perceived stress

Eating behavior

Intuitive eating

Emotional eating

Dietary intake

Obesity

\begin{abstract}
A B S T R A C T
Stress-related eating may be a potential factor in the obesity epidemic. Rather little is known about how stress associates with eating behavior and food intake in overweight individuals in a free-living situation. Thus, the present study aims to investigate this question in psychologically distressed overweight and obese working-aged Finns.

The study is a cross-sectional baseline analysis of a randomized controlled trial. Of the 339 study participants, those with all the needed data available ( $\mathrm{n}=297,84 \%$ females) were included. The mean age was $48.9 \mathrm{y}(\mathrm{SD}=7.6)$ and mean body mass index $31.3 \mathrm{~kg} / \mathrm{m}^{2}(\mathrm{SD}=3.0)$. Perceived stress and eating behavior were assessed by self-reported questionnaires Perceived Stress Scale (PSS), Intuitive Eating Scale, the Three-Factor Eating Questionnaire, Health and Taste Attitude Scales and ecSatter Inventory. Diet and alcohol consumption were assessed by 48-h dietary recall, Index of Diet Quality, and AUDIT-C.

Individuals reporting most perceived stress (i.e. in the highest PSS tertile) had less intuitive eating, more uncontrolled eating, and more emotional eating compared to those reporting less perceived stress $(\mathrm{p}<0.05)$. Moreover, individuals in the highest PSS tertile reported less cognitive restraint and less eating competence than those in the lowest tertile $(\mathrm{p}<0.05)$. Intake of whole grain products was the lowest among those in the highest PSS tertile $(\mathrm{p}<0.05)$. Otherwise the quality of diet and alcohol consumption did not differ among the PSS tertiles.

In conclusion, high perceived stress was associated with the features of eating behavior that could in turn contribute to difficulties in weight management. Stress-related way of eating could thus form a potential risk factor for obesity. More research is needed to develop efficient methods for clinicians to assist in handling stress-related eating in the treatment of obese people.
\end{abstract}

(c) 2016 Elsevier Ltd. All rights reserved.

\footnotetext{
* Corresponding author.

E-mail addresses: elina.jarvela-reijonen@uef.fi (E. Järvelä-Reijonen), leila. karhunen@uef.fi (L. Karhunen), essi.sairanen@jyu.fi (E. Sairanen), sanni.rantala@ helsinki.fi (S. Rantala), jaana.laitinen@ttl.fi (J. Laitinen), sampsa.puttonen@ttl.f (S. Puttonen), katri.peuhkuri@netnut.fi (K. Peuhkuri), maarit.hallikainen@uef.fi (M. Hallikainen), kristiina.juvonen@uef.fi (K. Juvonen), tero.myllymaki@firstbeat.fi (T. Myllymäki), tiina.fohr@jyu.fi (T. Föhr), jussi.pihlajamaki@uef.fi (J. Pihlajamäki), riitta.korpela@helsinki.fi (R. Korpela), miikka.ermes@vtt.fi (M. Ermes), raimo. lappalainen@jyu.fi_ (R. Lappalainen),_marjukka.kolehmainen@uef.fi (M. Kolehmainen)
}

\section{Introduction}

Several definitions of stress include the idea in which "environmental demands tax or exceed the adaptive capacity of an organism, resulting in psychological and biological changes that may place persons at risk for disease" (Cohen, Kessler, \& Gordon, 1997). Adaptation or coping response to stress changes behavior which is one of the mechanisms how stress can increase the risk (Cohen, 
Janicki-Deverts, \& Miller, 2007) for, for example, weight gain (Wardle, Chida, Gibson, Whitaker, \& Steptoe, 2011), metabolic syndrome (Räikkönen, Matthews, \& Kuller, 2007), and cardiovascular disease (Cohen et al., 2007). In addition, eating as a way of trying to cope with stress has been related to failure to maintain weight loss among formerly obese (Byrne, 2002). Rather little is known about the relationship between stress and the behavioral traits of eating. There is a need for research between eating behavior and naturally occurring stress instead of laboratorymeasured, artificially-induced, acute stress which may not resemble the situations people encounter in their daily life (Stewart-Knox, 2014; Torres \& Nowson, 2007).

In previous studies in real-life context, chronic stress has been associated with higher uncontrolled eating (Groesz et al., 2012; Nevanperä et al., 2012) and emotional eating (Nevanperä et al., 2012) in women. These features of eating behavior, uncontrolled and emotional eating, are related to unhealthy dietary patterns and negative health-related outcomes such as higher body mass index (BMI) (Konttinen, Männistö, Sarlio-Lähteenkorva, Silventoinen, \& Haukkala, 2010; Nevanperä et al., 2012; de Lauzon et al., 2004). Stress seems to have a negative impact also on food choices. Chronic stress has been associated with increased consumption of highly palatable foods in women (Groesz et al., 2012) and in both genders (Oliver \& Wardle, 1999). Higher perceived stress has also been associated with increased consumption of high-fat snacks and fast-food items in both genders (Barrington, Beresford, McGregor, \& White, 2014). Similarly, stress-driven eating, meaning eating or drinking to feel better in stressful situations, has been related to more frequent consumption of sausages, hamburgers, pizza, chocolate and alcoholic beverages in both men and women and adolescents (Jääskeläinen et al., 2014; Laitinen, Ek, \& Sovio, 2002). However, stress has not been consistently associated with the quality of diet (Barrington et al., 2014; Ferranti et al., 2013; Grossniklaus et al., 2010; Suominen-Taipale et al., 2010).

To the best of our knowledge, the association between stress and positive features of eating behavior such as intuitive eating (Tylka, 2006) and eating competence (Satter, 2007) has not been studied earlier. This association might be interesting since it has been observed that intuitive eating, e.g. eating based on hunger and satiety and not emotions, has been associated with lower BMI (Herbert, Blechert, Hautzinger, Matthias, \& Herbert, 2013; Tylka, 2006), better psychological well-being (Tylka, 2006), and possibly better dietary intake and/or eating behavior (Van Dyke \& Drinkwater, 2014). Eating competence, e.g. having positive attitudes about eating and consuming the amount of food that satiates hunger and appetite, has also been associated with lower BMI (Krall \& Lohse, 2011; Lohse, Satter, Horacek, Gebreselassie, \& Oakland, 2007), in addition to higher HDL-cholesterol and lower systolic blood pressure (Psota, Lohse, \& West, 2007), and better diet quality (Lohse, Bailey, Krall, Wall, \& Mitchell, 2012).

Improved understanding on eating behavior, food consumption and nutrient intake associated with perceived stress might help to develop methods and contents of dietary counseling and thus improve their effectiveness among overweight and obese individuals. However, to the best of our knowledge, the associations of eating behavior and diet quality with perceived stress have not been studied earlier in psychologically distressed overweight and obese adults in a naturalistic setting outside laboratory. Therefore, the aim of the present study was to investigate, in a real-life context, features of eating behavior, both unfavorable and favorable, food consumption, and energy nutrient intake in psychologically distressed overweight and obese Finns reporting different levels of perceived stress. We hypothesized that higher level of perceived stress associates with unfavorable eating behavior and poorer diet quality.

\section{Methods}

\subsection{Study design and participants}

The present paper is a cross-sectional retrospective analysis of the baseline measurements of the Elixir clinical intervention trial (Lappalainen et al., 2014). The aim of the Elixir study was to investigate the effect of three different psychological intervention methods on psychological and physiological well-being and on the features of metabolic syndrome. The study was approved by the ethics committee of the Central Finland Health Care District (reference number 7U/2012) and it was performed in accordance with the Declaration of Helsinki. The participants gave their written informed consent before participating. The study is registered with ClinicalTrials.gov with the identifier NCT01738256.

The study protocol is described by Lappalainen et al. (2014). Briefly, the study participants were recruited by advertisements in local newspapers. As the criterion, the participants had to be 25-60 years old and have a BMI $27-34.9 \mathrm{~kg} / \mathrm{m}^{2}$. They also had to be psychologically distressed $\geq 3$ points from the General Health Questionnaire, GHQ-12 (Goldberg, 1972), and have computer and internet access. There were also several exclusion criteria, such as diagnosed severe chronic illness and eating disorder (bulimia nervosa) evaluated at the screening and asked in the baseline questionnaire. The multicenter study was conducted in three cities in Finland (Jyväskylä, Kuopio and Helsinki). The study was carried out in two phases in order to diminish the effect of seasonal variation and because of the limited capacity to conduct measurements per day. The baseline measurements were taken in September and October 2012, and in January and February 2013. The participants filled in electronic questionnaires, visited the local study center for clinical and biochemical measurements, and reported their food consumption in a 48 -h dietary recall by telephone.

After randomization ( $\mathrm{n}=339), 41(12 \%)$ participants dropped out or were excluded (not fitting inclusion and exclusion criteria, $\mathrm{n}=19$ (6\%); medical condition, $\mathrm{n}=2(1 \%)$; not attending to the baseline measurements, $n=20(6 \%))$. In addition, one participant did not complete all the questionnaires. Therefore, 297 participants (249 females, 48 males) were included for the analysis of the present study. The descriptive characteristics of the participants are shown in Table 1. Majority (79\%) had at least college education. Most of the participants (84\%) did not smoke. Almost three-fourths (74\%) were married or cohabiting, and $41 \%$ had one or more underage children. Majority (89\%) was aiming to lose weight.

\subsection{Measurements}

\subsubsection{Clinical measurements}

Weight, height and waist circumference were measured at each study center in the morning after a 12-h overnight fast with calibrated instruments (Lappalainen et al., 2014). BMI was calculated

Table 1

Descriptive characteristics of the participants $(n=297)$.

\begin{tabular}{lcl}
\hline Characteristic & Mean $\pm \mathrm{SD}$ & Range \\
\hline Age $(\mathrm{y})$ & $48.9 \pm 7.6$ & $26.6-60.8$ \\
Weight $(\mathrm{kg})$ & $87.9 \pm 11.0$ & $64.0-120.1$ \\
Height $(\mathrm{cm})$ & $167.5 \pm 8.0$ & $149.0-195.6$ \\
BMI $\left(\mathrm{kg} / \mathrm{m}^{2}\right)$ & $31.3 \pm 3.0$ & $25.3-40.1$ \\
Waist circumference $(\mathrm{cm})$ & $102.9 \pm 8.7$ & $79.7-124.0$ \\
Psychological distress & $7.1 \pm 2.8$ & $3.0-12.0$ \\
(GHQ-12 score) $^{\mathrm{a}}$ & & \\
\hline
\end{tabular}

Notes: BMI = body mass index; GHQ-12 = General Health Questionnaire-12. a Bimodal scale. 
from the measured weight and height as kilograms per meters squared.

\subsubsection{Background characteristics}

Demographic information, such as age, marital status, number of underage children in household, education, smoking, regular medication, and type of working hours (daytime job, two-shift work, or irregular work), was collected by a questionnaire. Symptoms of depression were measured by the Beck Depression Inventory-II (Beck, Steer, Ball, \& Ranieri, 1996). Depressive symptoms were categorized into no/minimal depression (0-13 points), mild depression (14-19 points), moderate depression (20-28 points), and severe depression (29-63 points).

\subsubsection{Psychological distress and perceived stress}

The 12-item General Health Questionnaire, GHQ-12 (Goldberg, 1972), was used for screening the volunteers for psychological distress. It has been found to be a valid screening tool for common mental health problems also in the Finnish population (Holi, Marttunen, \& Aalberg, 2003). Respondent was asked, considering the past few weeks, to answer questions such as "Have you recently felt capable of making decisions about things?". In the screening, bimodal GHQ scoring was used: "more so than usual" (0 points); "same as usual" (0); "less so than usual" (1); and "much less capable" (1), with the total sum score ranging from 0 to 12 . Cronbach's alpha using bimodal scoring was 0.72 . In the present analyses, Likert scoring system $(0,1,2,3$ points; possible range $0-36)$ was used for statistical analyses to achieve larger variation of the GHQ-12 scores. Cronbach's alpha using Likert scoring was 0.80 .

Perceived Stress Scale, PSS (Cohen, Kamarck, \& Mermelstein, 1983), is a 14-item measure for assessing the degree to which a person perceive life as stressful. The questionnaire has proved acceptable psychometric properties worldwide (Lee, 2012). Questions concern how often a person has experienced certain feelings and thoughts during the previous month, for example "In the last month, how often have you found that you could not cope with all the things that you had to do?". The 5-point Likert scale from "never" (0) to "very often" (4) is summed for the total score (possible range $0-56$ ). Half of the items are positive, for example "In the last month, how often have you felt that things were going your way?". Those items are scored in the reverse direction. Cronbach's alpha was 0.88 in this sample.

\subsubsection{Eating behavior}

Intuitive Eating Scale, IES (Tylka, 2006), consists of 21 items with subcategories of the intuitive eating: (a) Unconditional Permission to Eat ( 9 items, for example "If I am craving a certain food, I allow myself to have it."), (b) Eating for Physical Rather Than Emotional Reasons (6 items, for example "I stop eating when I feel full (not overstuffed)."), and (c) Reliance on Internal Hunger/Satiety Cues (6 items, for example "I trust my body to tell me when to eat."). The statements are answered with a 5-point Likert scale ranging from "strongly disagree" (1) to "strongly agree" (5). The scores are averaged, thus possible ranges of IES total score and its subscales are $1-5$. Cronbach's alpha was 0.80 for the whole scale and 0.69 ; 0.86; 0.76 for the subscales Unconditional Permission to Eat, Eating for Physical Rather Than Emotional Reasons, and Reliance on Internal Hunger/Satiety Cues, respectively.

The Three-Factor Eating Questionnaire, TFEQ-R18 (Karlsson, Persson, Sjöström, \& Sullivan, 2000), was used to measure (a) Cognitive Restraint ( 6 items, for example "I deliberately take small helpings as a means of controlling my weight."), (b) Uncontrolled Eating (9 items, for example "Sometimes when I start eating, I just can't seem to stop."), and (c) Emotional Eating (3 items, for example "When I feel blue, I often overeat."). Answers are given by 4-point
Likert scale except for one item which is answered by 8-point Likert scale. The possible range of the total scores is $0-100$. Cronbach's alphas were $0.70 ; 0.88 ; 0.88$ for the subscales Cognitive Restraint, Uncontrolled Eating, and Emotional Eating, respectively.

Of the Finnish Health and Taste Attitude Scales, HTAS (Roininen, Lähteenmäki, \& Tuorila, 1999), subcategories (a) Pleasure (6 items, for example "When I eat, I concentrate on enjoying the taste of food.") and (b) Using Food as a Reward (6 items, for example "I reward myself by buying something really tasty.") were used. The statements were answered with a 7-point Likert scale ranging from "strongly disagree" (1) to "strongly agree" (7). The scores are averaged, thus the possible ranges of the scores are $1-7$. Cronbach's alphas were 0.71 and 0.79 for the subcategories Pleasure and Using Food as a Reward, respectively.

Eating competence was measured by preliminary Finnish translation of ecSatter Inventory for Low-Income, ecSI/LI (Krall \& Lohse, 2010, 2011) which was recently named as ecSI 2.0 (Lohse, 2015). The definition of eating competence consists of four components which also constitutes the 16-item questionnaire's subcategories: (a) Eating Attitudes ( 5 items, for example "I am relaxed about eating."), (b) Food Acceptance (3 items, for example "I experiment with new food and learn to like it."), (c) Internal Regulation (3 items, for example "I eat as much as I am hungry for."), and (d) Contextual Skills (5 items, for example "I generally plan for feeding myself. I don't just grab food when I get hungry."). The statements are answered: "always" (3 points); "often" (2); "sometimes" (1); "rarely" (0); or "never" $(0)$. The possible range of Eating Competence total sum score is 0-48; Eating Attitudes and Contextual Skills 0-15; and Food Acceptance and Internal Regulation 0-9. Total sum score $\geq 32$ indicates a competent eater (Lohse et al., 2007) meaning that the person has positive attitudes about eating and about food, accepts and eats an ever-increasing variety of foods, eats intuitively enough according to internal hunger and satiety signals, and has skills and resources for managing daily meals (Satter, 2007). Cronbach's alpha was 0.76 for the whole scale and $0.62 ; 0.66 ; 0.58 ; 0.74$ for the subscales Eating Attitudes, Food Acceptance, Internal Regulation, and Contextual Skills, respectively.

\subsubsection{Food consumption and nutrient intake}

A concise measure of food consumption, Index of Diet Quality (IDQ) (Leppälä, Lagström, Kaljonen, \& Laitinen, 2010), consists of 18 questions about frequency, portion size and type of certain foods and drinks consumed during the previous month to evaluate adherence to nutrition recommendations. It has been developed and validated in Finnish healthy, mostly normal weight, adult females by using the seven-day food record (Leppälä et al., 2010). The possible IDQ total score is $0-15$. Points below 10 indicate nonadherence and points from 10 to 15 indicate adherence to the health-promoting diet (Leppälä et al., 2010). In this study, answers that seemed possibly unrealistic or outliers were checked from the participant and corrections were made when needed. Despite this procedure, one answer ( 10 glasses $=20 \mathrm{dl}$ juice per day) remained unverified and was coded as missing.

Alcohol consumption during the previous six months was measured by the Finnish version of the questionnaire Alcohol Use Disorders Identification Test Consumption, AUDIT-C (Bush, Kivlahan, McDonell, Fihn, \& Bradley, 1998). It has been shown to have strong correlation to alcohol consumption in a general Finnish population (Aalto, Alho, Halme, \& Seppä, 2009). The questionnaire contains three questions about the frequency and amounts of alcohol usage. For the questions concerning the amount of drinks consumed, a list of typical Finnish serving sizes and their corresponding amounts as standard drinks (e.g. $33 \mathrm{cl}$ bottle of beer is one drink) were provided. The responses were scored from 0 to 4 and summed, and the possible total score is from 0 to 12 . Cronbach's 
alpha was 0.69 .

The 48-h dietary recall was conducted to collect information of nutrient intake. Participants were asked to describe all foods and drinks eaten and drunk during the previous full $48 \mathrm{~h}$ (from 00.00 to 24.00). The interview was conducted by trained nutritionists on the telephone at pre-scheduled time. Participants were told that the interview considers diet but anything about 48-h recall was not mentioned beforehand. An electronic picture book (Paturi, Nieminen, Reinivuo, \& Ovaskainen, 2006) was used to help to describe portion sizes. The interviews were performed from Tuesday to Friday, so Fridays and Saturdays are excluded from the data. The collected data was coded and nutrient intake calculated with AivoDiet software version 2.0.2.2 (Aivo Ltd., Turku, Finland) using the Fineli ${ }^{\circledR}$ Finnish Food Composition Database (National Institute for Health and Welfare, Nutrition Unit, Helsinki, Finland). The interview protocol of the 48-h dietary recall was created based on the face-to-face 48-h dietary recall conducted in the national FINDIET 2012 survey (Helldán et al., 2013). The 48-h dietary recall protocol of the Elixir study was done together by the three nutritionists who also conducted the interviews. The participants were encouraged to be truthful in the 48-h dietary recall and they were told that the interviewer will not assess or comment on their eating and drinking or give any dietary guidance. The foods and beverages consumed during the $48 \mathrm{~h}$ were repeated at the end and the interviewer encouraged the participant to make additions or modifications while repeating the course of days' events.

Data collected by the 48-h dietary recall was also utilized to calculate average daily intake of food items in specific categories: Vegetables (excluding potatoes); Fruits and berries; Rye bread; Whole grain products; Fish; Pastries (sweet and savory); and Salty snacks and sweets. Food consumption was calculated as grams and energy-adjusted grams per $1 \mathrm{MJ}$.

\subsubsection{Statistical methods}

Data were analyzed using IBM SPSS Statistics version 21. The study participants were divided into tertiles based on their PSS sum scores; the lowest $(\mathrm{n}=104$, mean $=18.3, \mathrm{SD}=4.2$, range $=7-23$ ), middle $(\mathrm{n}=102$, mean $=27.0, \mathrm{SD}=1.9$, range $=24-30)$, and the highest $(\mathrm{n}=91$, mean $=35.4, \mathrm{SD}=3.8$, range $=31-52)$ tertile because there are no cut-off scores for the scale (Cohen, 2015). The normality assumption was assessed by the histograms of the standardized residuals. Intake of sucrose (E\%) was log-transformed to achieve normal distribution of the standardized residuals. All the food consumption variables ( $\mathrm{g}$ and $\mathrm{g} / \mathrm{MJ}$ ) were non-normally distributed.

To test the differences of normally distributed continuous variables in PSS tertiles, one-way ANOVA with Tukey HSD post hoc comparisons was used. Multiway ANOVAs were conducted by adjusting for study center, starting time of the study, aim to lose weight, and gender. Non-normally distributed variables were analyzed in PSS tertiles by Kruskall-Wallis test. Categorical variables in PSS tertiles were assessed by Pearson chi-square test or Fisher's exact test.

Pearson correlation was used to assess the relation of continuous normally distributed variables and Spearman correlation for non-normally distributed variables. P-values $<0.05$ were considered as statistically significant.

\section{Results}

\subsection{Psychological distress and perceived stress}

In the whole study population the mean GHQ-12 Likert score was $19.5 \pm 4.3$ (mean \pm SD) and the scores ranged from 10 to 34 . PSS was positively correlated to GHQ-12 Likert score ( $r=0.461$, $\mathrm{p}<0.001)$. Although psychologically distressed participants were recruited, there was a large variation in perceived stress among the participants: PSS scores ranged from 7 to 52 with the mean $26.5 \pm 7.7$.

The participants were evenly distributed in the PSS tertiles in terms of their descriptive characteristics. There were no statistically significant differences among PSS tertiles in starting time of the study, study center, gender, age, BMI, waist circumference, marital status, number of underage children in household, education level, smoking, having regular medication, and type of working hours (Table 2). Aiming to lose weight was more common in the lowest and middle PSS tertiles than in the highest tertile $\left(\chi^{2}(2)=6.837\right.$, $\mathrm{p}=0.033$ ) and depressive symptoms were more common and severe in the highest tertile than in the lowest or middle tertiles $(\mathrm{p}<0.001)$.

\subsection{Association of eating behavior with perceived stress}

Higher perceived stress was associated with several features of eating behavior: lower intuitive eating $(F[2,294]=5.49, \mathrm{p}=0.005)$, less eating for physical rather than emotional reasons (F[2, $294]=8.52, \mathrm{p}<0.001)$, lower reliance on internal hunger/satiety cues $(\mathrm{F}[2,294]=3.84, \mathrm{p}=0.023)$, less cognitive restraint $(\mathrm{F}[2$, $294]=3.72, p=0.025)$, lower eating competence $(F[2,294]=4.63$, $\mathrm{p}=0.011)$, and lower contextual skills $(\mathrm{F}[2,294]=5.77, \mathrm{p}=0.003)$ (Fig. 1). In addition, higher perceived stress was associated with uncontrolled eating $(\mathrm{F}[2,294]=9.18, \mathrm{p}<0.001)$, emotional eating $(\mathrm{F}[2,294]=11.69, \mathrm{p}<0.001)$, seeking pleasure from food $(\mathrm{F}[2$, $294]=3.08, \mathrm{p}=0.047)$, and using food as a reward $(F[2$, $294]=4.73, \mathrm{p}=0.010)$. Post hoc analyses indicated that compared

Table 2

Descriptive characteristics of the participants $(n=297)$ in the perceived stress tertiles.

\begin{tabular}{|c|c|c|c|c|}
\hline \multirow[t]{2}{*}{ Characteristic } & \multicolumn{3}{|l|}{ PSS tertiles } & \multirow[t]{2}{*}{$p$-value } \\
\hline & $\begin{array}{l}\text { Lowest } \\
(7-23) \\
(n=104)\end{array}$ & $\begin{array}{l}\text { Middle } \\
(24-30) \\
(\mathrm{n}=102)\end{array}$ & $\begin{array}{l}\text { Highest } \\
(31-52) \\
(\mathrm{n}=91)\end{array}$ & \\
\hline \multicolumn{5}{|l|}{ Starting time of the study $(\mathrm{n})$} \\
\hline Autumn & 50 & 43 & 49 & \\
\hline Spring & 54 & 59 & 42 & 0.267 \\
\hline \multicolumn{5}{|l|}{ Study center $(\mathrm{n})$} \\
\hline Jyväskylä & 25 & 31 & 27 & \\
\hline Kuopio & 41 & 29 & 26 & \\
\hline Helsinki & 38 & 42 & 38 & 0.435 \\
\hline \multicolumn{5}{|l|}{ Gender ( $\mathrm{n})$} \\
\hline Female & 87 & 85 & 77 & \\
\hline Male & 17 & 17 & 14 & 0.969 \\
\hline Age (y) & $48.9 \pm 7.8$ & $49.9 \pm 7.4$ & $47.8 \pm 7.6$ & 0.163 \\
\hline BMI $\left(\mathrm{kg} / \mathrm{m}^{2}\right)$ & $31.5 \pm 2.8$ & $31.1 \pm 3.2$ & $31.4 \pm 3.1$ & 0.577 \\
\hline Waist circumference $(\mathrm{cm})$ & $102.3 \pm 8.1$ & $103.4 \pm 9.5$ & $102.9 \pm 8.6$ & 0.674 \\
\hline \multicolumn{5}{|c|}{ Aim to lose weight (n) } \\
\hline Yes & 96 & 93 & 74 & \\
\hline No & 8 & 9 & 17 & 0.033 \\
\hline \multicolumn{5}{|l|}{ Depression (BDI-II) (n) } \\
\hline No/minimal & 85 & 56 & 20 & \\
\hline Mild & 17 & 37 & 27 & \\
\hline Moderate & 2 & 9 & 37 & \\
\hline Severe & 0 & 0 & 7 & $<0.001$ \\
\hline \multicolumn{5}{|l|}{ Marital status (n) } \\
\hline Married/cohabiting & 84 & 76 & 61 & \\
\hline Other $^{\mathrm{b}}$ & 20 & 26 & 30 & 0.090 \\
\hline
\end{tabular}

Notes: Values are $\mathrm{n} /$ mean $\pm \mathrm{SD}$; PSS = Perceived Stress Scale (possible score range 0-56); Autumn = September-October; Spring = January-February; BMI = body mass index; BDI-II = The Beck Depression Inventory-II.

a p-value for differences between the perceived stress tertiles (Pearson chi-square or Fisher's Exact test for categorical variables and one-way ANOVA for continuous variables).

b Unmarried, estranged, divorced or widow/widower. 

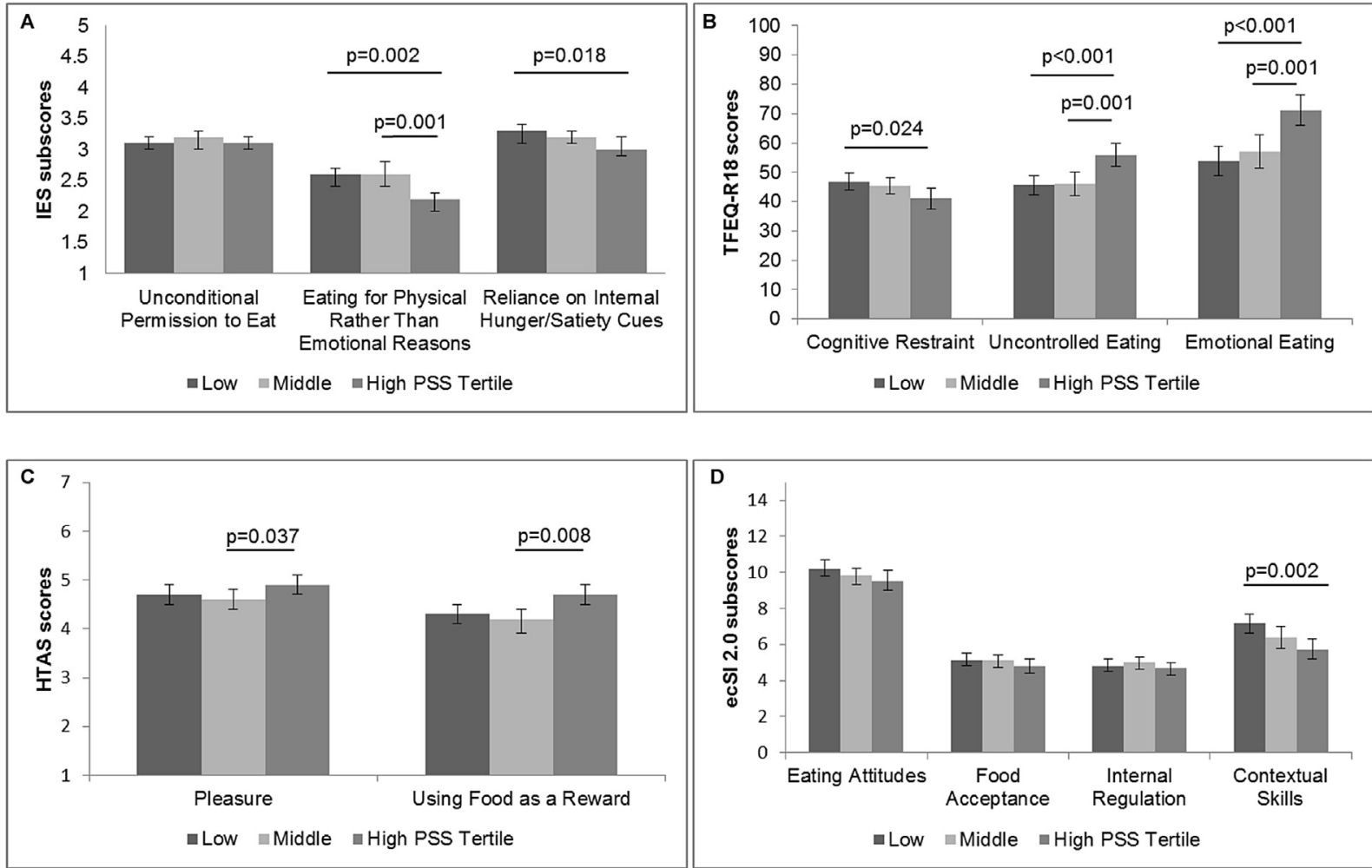

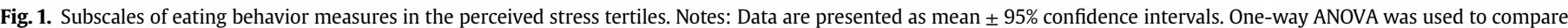

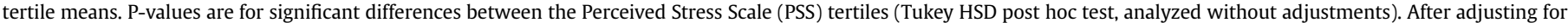

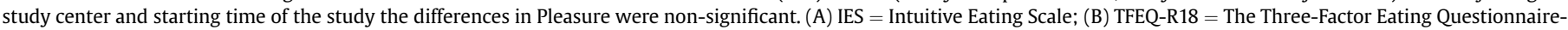

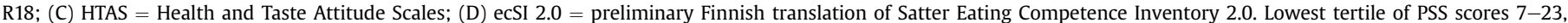
$\mathrm{n}=104$, middle tertile of PSS scores $24-30, \mathrm{n}=102$, and highest tertile of PSS scores $31-52, \mathrm{n}=91$.

to the middle and lowest PSS tertiles, persons in the highest tertile (i.e. reporting most perceived stress) had less intuitive eating (mean \pm SD $30.0 \pm 0.5$ vs. $30.0 \pm 0.5$ vs. $2.8 \pm 0.5$, Tukey HSD post hoc test, $\mathrm{p}=0.007, \mathrm{p}=0.019$, respectively). Furthermore, eating competence was significantly lower in the highest compared to the lowest perceived stress tertile (mean \pm SD $24.7 \pm 5.7$ vs. $27.3 \pm 6.1$, Tukey HSD post hoc test, $\mathrm{p}=0.007)$. Other post hoc test results are shown in Fig. 1. The associations remained statistically significant after adjusting for gender.

After adjustment for study center and starting time of the study, the association of perceived stress with seeking pleasure from food was not statistically significant $(F[2,287]=1.74, \mathrm{p}=0.178)$ any more. Adjusting further for aim to lose weight, the associations of perceived stress with seeking pleasure from food $(F[2,286]=1.75$, $\mathrm{p}=0.175)$ and cognitive restraint $(\mathrm{F}[2,290]=2.41, \mathrm{p}=0.092)$ were not statistically significant any more.

Fifty-eight (20\%) participants were competent eaters (ecSI 2.0 total score $\geq 32$ ). The difference in the proportion of competent eaters in PSS tertiles was statistically significant $\left(\chi^{2}(2)=7.764\right.$, $\mathrm{p}=0.021)$ : nine $(16 \%)$ of the competent eaters were in the highest tertile of perceived stress whereas of the non-competent eaters there were $34 \%(n=82)$.

\subsection{Associations of food consumption and nutrient intake with perceived stress}

There were no significant differences among the tertiles of perceived stress in energy nutrient intake or alcohol use. A trend $(p=0.076)$ was observed for lower IDQ score in the highest tertile of perceived stress, but it did not remain after adjustment for study center and starting time of the study (Table 3). After adjustment for study center and starting time of the study, the association of perceived stress with the percentage of energy from saturated fat became statistically significant $(F[2,287]=3.50, p=0.032)$ but no statistically significant differences between the tertiles were found in the post hoc analysis. Adjusting further for gender or aim to lose weight, no statistically significant associations were found.

Most participants $(n=193,65 \%)$ reported high adherence to the health-promoting diet (IDQ score $\geq 10$ ). The adherence to the health-promoting diet did not differ significantly among PSS tertiles $69 \%$ in the lowest, $67 \%$ in the middle, and $59 \%$ in the highest PSS tertile, $\chi^{2}[2, \mathrm{~N}=296]=2.106, \mathrm{p}=0.349$ ).

Intake of whole grain products as grams and energy-adjusted grams per megajoule was lower in the most stressed participants than in the less stressed participants $(\mathrm{p}=0.040$ and $\mathrm{p}=0.013$, respectively) (Table 4). There were no differences in the intake of vegetables, fruits and berries, rye bread, fish, sweet and savory pastries, and salty snacks and sweets among the PSS tertiles. Nevertheless, there was a tendency $(p=0.067)$ for lower energyadjusted intake of vegetables, fruits and berries in the highest PSS tertile than in the middle and the lowest tertiles (Table 4).

\subsection{Associations of eating behavior, energy intake and perceived stress}

As an additional information, the association of energy intake and perceived stress was analyzed according to the participants' ratings of stress-eating. Intuitive Eating Scale (IES) includes an item directly related to stress "I find myself eating when I am stressed out, even when I'm not physically hungry". Most of the participants 
Table 3

Diet quality, alcohol use and intake of energy nutrients in the perceived stress tertiles.

\begin{tabular}{|c|c|c|c|c|c|}
\hline & \multicolumn{3}{|l|}{ PSS tertiles } & \multirow[t]{2}{*}{$p$-value ${ }^{a}$} & \multirow[t]{2}{*}{$p$-value ${ }^{b}$} \\
\hline & $\begin{array}{l}\text { Lowest } \\
(7-23) \\
(n=104)\end{array}$ & $\begin{array}{l}\text { Middle } \\
(24-30) \\
(\mathrm{n}=102)\end{array}$ & $\begin{array}{l}\text { Highest } \\
(31-52) \\
(\mathrm{n}=91)\end{array}$ & & \\
\hline \multicolumn{6}{|l|}{ Diet quality and alcohol use } \\
\hline IDQ score & $10.4 \pm 1.9^{c}$ & $10.3 \pm 2.1$ & $9.8 \pm 2.3$ & 0.076 & 0.119 \\
\hline AUDIT-C score & $3.6 \pm 2.1$ & $4.0 \pm 2.6$ & $4.0 \pm 2.3$ & 0.374 & 0.366 \\
\hline \multicolumn{6}{|l|}{ Intake of energy nutrients } \\
\hline Energy (kJ) & $8040 \pm 2326$ & $8289 \pm 2520$ & $8456 \pm 2231$ & 0.466 & 0.397 \\
\hline Protein (E\%) & $17.3 \pm 3.5$ & $17.9 \pm 4.2$ & $17.1 \pm 4.5$ & 0.336 & 0.488 \\
\hline Carbohydrate (E\%) & $41.5 \pm 7.2$ & $40.7 \pm 7.9$ & $40.3 \pm 7.6$ & 0.507 & 0.602 \\
\hline Total fat (E\%) & $37.3 \pm 7.6$ & $37.1 \pm 8.4$ & $38.2 \pm 7.6$ & 0.564 & 0.609 \\
\hline Saturated fat (E\%) & $13.2 \pm 3.4$ & $13.3 \pm 3.7$ & $14.3 \pm 4.3$ & 0.079 & 0.032 \\
\hline Monounsaturated fat (E\%) & $13.1 \pm 3.6$ & $12.6 \pm 3.4$ & $13.0 \pm 3.0$ & 0.521 & 0.536 \\
\hline Polyunsaturated fat (E\%) & $6.3 \pm 1.9$ & $6.4 \pm 2.1$ & $6.5 \pm 2.3$ & 0.804 & 0.704 \\
\hline Sucrose (E\%) & $9.6 \pm 4.3$ & $8.7 \pm 5.1$ & $9.1 \pm 4.6$ & 0.160 & 0.191 \\
\hline Fiber $(\mathrm{g})$ & $22.5 \pm 8.2$ & $23.9 \pm 10.1$ & $22.0 \pm 9.4$ & 0.292 & 0.402 \\
\hline Fiber (g/MJ) & $2.9 \pm 1.0$ & $2.9 \pm 1.0$ & $2.6 \pm 1.0$ & 0.093 & 0.131 \\
\hline
\end{tabular}

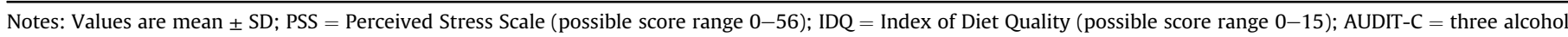
consumption questions from the Alcohol Use Disorders Identification Test (possible score range 0-12); E\% = percentage of energy.

${ }^{a}$ p-value for differences between the perceived stress tertiles (one-way ANOVA).

b p-value adjusted for study center and starting time (three-way independent ANOVA)

${ }^{c} \mathrm{n}=103$ because of one missing value in IDQ score.

Table 4

Food consumption as grams and energy-adjusted grams per $\mathrm{MJ}$ in the perceived stress tertiles.

\begin{tabular}{|c|c|c|c|c|}
\hline & \multicolumn{3}{|l|}{ PSS tertiles } & \multirow[t]{3}{*}{$p$-value } \\
\hline & $\begin{array}{l}\text { Lowest } \\
(7-23) \\
(\mathrm{n}=104)\end{array}$ & $\begin{array}{l}\text { Middle } \\
(24-30) \\
(\mathrm{n}=102)\end{array}$ & $\begin{array}{l}\text { Highest } \\
(31-52) \\
(\mathrm{n}=91)\end{array}$ & \\
\hline & \multicolumn{3}{|l|}{ Mean $(95 \% \mathrm{CI})$} & \\
\hline \multicolumn{5}{|l|}{ Vegetables } \\
\hline g/day & $205(183-227)$ & $192(170-215)$ & $190(163-217)$ & 0.423 \\
\hline $\mathrm{g} / \mathrm{MJ} /$ day & $27(24-30)$ & $24(21-27)$ & $24(20-27)$ & 0.167 \\
\hline \multicolumn{5}{|c|}{ Fruits and berries } \\
\hline g/day & $256(215-297)$ & $272(216-329)$ & $223(180-267)$ & 0.391 \\
\hline $\mathrm{g} / \mathrm{MJ} /$ day & $34(28-40)$ & $35(28-43)$ & $27(22-32)$ & 0.214 \\
\hline \multicolumn{5}{|c|}{ Vegetables, fruits and berries } \\
\hline g/day & $461(414-509)$ & $465(403-527)$ & $413(357-470)$ & 0.269 \\
\hline $\mathrm{g} / \mathrm{MJ} / \mathrm{day}$ & $61(54-68)$ & $59(51-67)$ & $50(44-57)$ & 0.067 \\
\hline \multicolumn{5}{|l|}{ Rye bread } \\
\hline g/day & $55(44-66)$ & $63(52-73)$ & $57(47-67)$ & 0.359 \\
\hline $\mathrm{g} / \mathrm{MJ} / \mathrm{day}$ & $7(6-8)$ & $8(6-9)$ & $7(6-8)$ & 0.370 \\
\hline \multicolumn{5}{|c|}{ Whole grain products } \\
\hline g/day & $141(119-164)$ & $151(127-174)$ & $114(91-136)$ & 0.040 \\
\hline $\mathrm{g} / \mathrm{MJ} /$ day & $18(15-20)$ & $19(16-22)$ & $14(11-16)$ & 0.013 \\
\hline \multicolumn{5}{|l|}{ Fish } \\
\hline g/day & $28(20-35)$ & $24(16-32)$ & $27(18-35)$ & 0.677 \\
\hline $\mathrm{g} / \mathrm{MJ} /$ day & $4(3-4)$ & $3(2-4)$ & $3(2-4)$ & 0.625 \\
\hline \multicolumn{5}{|c|}{ Pastries (sweet and savory) } \\
\hline g/day & $67(52-82)$ & $88(63-113)$ & $86(64-107)$ & 0.370 \\
\hline $\mathrm{g} / \mathrm{MJ} /$ day & $8(6-10)$ & $10(8-12)$ & $9(7-11)$ & 0.364 \\
\hline \multicolumn{5}{|c|}{ Salty snacks and sweets } \\
\hline g/day & $24(17-30)$ & $19(13-24)$ & $28(20-36)$ & 0.515 \\
\hline $\mathrm{g} / \mathrm{MJ} /$ day & $3(2-4)$ & $2(2-3)$ & $3(2-4)$ & 0.536 \\
\hline
\end{tabular}

Notes: Values are average of two days consumption based on 48-h dietary recall. PSS $=$ Perceived Stress Scale (possible score range 0-56); $\mathrm{CI}=$ confidence interval for mean.

${ }^{a}$ p-value for differences between the perceived stress tertiles (Kruskall-Wallis test).

strongly agreed (24\%) or agreed (49\%) with the statement whereas $11 \%$ disagreed and $5 \%$ strongly disagreed. These IES responses were associated with perceived stress $\left(\chi^{2}(8)=21.507, \mathrm{p}=0.006\right)$ so that eating during stress was more common in the highest PSS tertile compared to the middle and the lowest tertiles. These IES responses were, however, not associated with actual energy intake in general
$(\mathrm{F}[4,292]=0.365, \mathrm{p}=0.834)$ or separately in the PSS tertiles $(\mathrm{F}[4$, $99]=0.258, \mathrm{p}=0.904 ; \mathrm{F}[4,97]=0.090, \mathrm{p}=0.985$; and $\mathrm{F}[4$, $86]=0.123, \mathrm{p}=0.974$ in the lowest, middle and highest tertile, respectively).

\section{Discussion}

In the present study we found that higher level of perceived stress was associated with lower intuitive eating, lower eating competence and lower cognitive restraint, higher uncontrolled and emotional eating, and higher tendency to use food as a reward in psychologically distressed overweight and obese working-age Finns examined in a naturalistic setting. This was the first time, to the best of our knowledge, that favorable characteristics of eating behavior, intuitive eating and eating competence, were shown to be rare among overweight and obese adults with high perceived stress. The hypothesized association of perceived stress with poorer diet quality was not confirmed although lower consumption of whole grain products among the most stressed participants was found.

Consistently with our results, previous studies have found association between chronic or perceived stress and higher uncontrolled (Groesz et al., 2012; Nevanperä et al., 2012; Richardson, Arsenault, Cates, \& Muth, 2015) and emotional eating (Nevanperä et al., 2012; Richardson et al., 2015) in women. In the present study of perceived stress the association was observed regardless of gender. In addition to previous studies, we found that higher tendency to use food as a reward and seeking pleasure from food were associated with higher perceived stress, although the latter association disappeared after adjusting with items related to study design (i.e. study center, starting time of the study). This suggests that seeking pleasure from food at the different levels of perceived stress was different between participants in three study centers and/or between participants who answered the questionnaire in September-October and participants who answered it in January-February. Lower cognitive restraint was associated with higher level of perceived stress, but after adjusting for aiming to lose weight in addition to study center and starting time of the study, the association did not remain significant. This suggests that cognitive restraint was different at the different levels of perceived 
stress depending on whether a person is aiming to lose weight or not. This was logical, since aim of losing or maintaining weight is, by definition, associated with cognitive restraint of eating (de Lauzon et al., 2004) and the study participants with the highest level of perceived stress were also less aiming to lose weight.

In addition to being associated with the unfavorable features of eating behavior, higher level of perceived stress was inversely associated with the favorable features of eating behavior, intuitive eating and eating competence. Individuals with high perceived stress were less likely to eat and drink according to their inner feelings of hunger and satiety. Instead, their eating was more triggered by emotions and external cues. In addition, lower contextual skills among those with the highest level of perceived stress indicate irregular meal timing and planning for meals and less concentrating on eating. This could, in turn, be a risk factor for uncontrolled eating and less successful weight management.

By using the cut-off value set in earlier studies (Lohse et al., 2007), minority (20\%) of the participants were competent eaters (having positive attitude towards eating and food, being willing to eat variety of foods, consuming intuitively enough food, and manage daily meals (Satter, 2007)). The prevalence of competent eaters was even smaller among the most stressed participants (10\%). The proportion of competent eaters was clearly lower than the prevalence of $29-46 \%$ found in previous studies in adult populations not specifically targeted to psychologically distressed people (Krall \& Lohse, 2010, 2011; Lohse et al., 2007; Psota et al., 2007). It could thus be speculated that either perceived stress reduces eating competence or, vice versa, those with poor eating competence are more vulnerable to experience perceived stress. Moreover, the most stressed study participants had the lowest eating competence but the highest scores in emotional eating. It could thus be that eating is used for coping with negative feelings caused by stress, and the emotional eating could thus be a moderator between perceived stress and eating competence. However, these suggestions must be done with caution because of the cross-sectional study design as well as because the preliminary Finnish translation of ecSI 2.0 used in this study has not been validated in overweight and obese adults.

Our hypothesis that high perceived stress would be related to poorer diet was not confirmed. Higher perceived stress was associated to a significant degree only with lower whole grain consumption. This association has not been reported earlier. However, whole grain has been a part of an index or other definition of "healthy diet" in a few previous studies investigating the association between stress and diet (Ferranti et al. 2013; Groesz et al. 2012; Richardson et al. 2015). Persons with the highest perceived stress in the present study showed, in addition, a tendency towards lower diet quality, higher percentage of energy from saturated fat, and lower vegetable, fruit and berry consumption than persons with lower perceived stress.

Although we found clear association between perceived stress and uncontrolled and emotional eating, which, in turn, have been associated with non-beneficial food choices (Keskitalo et al., 2008; Konttinen et al., 2010) and higher energy intake (de Lauzon et al., 2004), we found no marked associations between perceived stress and food consumption or energy nutrient intake. This finding may be due to several possible reasons. The overall dietary quality during the past month was measured by a short questionnaire, and actual food and nutrient intake by a 48 -h dietary recall. It may be possible that these measures were incapable to detect the outcomes of certain features of eating behavior. For example, emotional eating may lead to overeating (Wallis \& Hetherington, 2009), but those overeating occasions may have not occurred during the recorded $48 \mathrm{~h}$. Furthermore, Fridays and Saturdays were not included to the interviews which may have influenced our results, as energy intake typically increases on weekends (Ocké et al., 2009; Racette et al., 2008). Long-term stress among working-age people may increase eating after the workweek, on Friday evenings and Saturdays. This stress-related overeating may be compensated by eating less or paying attention to quality of food during weekdays or in the beginning of the working week (Racette et al., 2008), which could explain our findings. Our results anyhow show that the possible stress-driven eating does not have at least a clear, direct influence on food consumption during weekdays. To the best of our knowledge, the weekday-weekend fluctuation has not been studied related to stress-driven eating. Some indication of this could however be seen in two earlier studies with students, in which no differences were observed between weekdays and weekend days in the association between perceived stress and hunger (Huh, Shiyko, Keller, Dunton, \& Schembre, 2015) or between the number of daily hassles and the number of snacks consumed (Conner, Fitter, \& Fletcher, 1999).

When measuring dietary intake, individuals tend to overestimate the intake of health-beneficial foods and underestimate the non-beneficial foods. Especially obese persons are shown to underreport the use of snack-type foods (Heitmann \& Lissner, 1995) which particularly have been reported to be consumed under stress (Groesz et al., 2012; Laitinen et al., 2002; Oliver \& Wardle, 1999). In statistical analyses also energy adjusted variables were used in order to try to handle underreporting. The possible misreporting was noticed already in the creating of the 48-h dietary recall interview protocol. Therefore, during the interview participants were encouraged to be truthful, the situation was aimed to be confidential and non-judgmental, and there were several possibilities for the interviewee to make changes to the record. An electronic picture book was used to help estimate portion sizes and record them in a standardized way. Total avoidance of misreporting food intake is challenging and would require more sophisticated methods such as continuous food intake monitoring by e.g. a wearable camera or analyzing nutritional biomarkers from e.g. urine or blood sample. In the present study, the high intake of vegetables, fruits, and berries (mean $>400 \mathrm{~g} /$ day in each PSS tertile) measured by the 48 -h dietary recall and high adherence (65\%) to the health-promoting diet measured by IDQ questionnaire suggest that there could have been especially overreporting of healthbeneficial foods. Furthermore, only $20 \%$ were competent eaters, a classification which has been associated with higher intake of fiber, several vitamins and minerals, and Healthy Eating Index (Lohse et al., 2012) which also refers to potential overreporting. On the other hand, this could reflect the fact that majority (89\%) of the participants reported they were aiming to lose weight. In the whole sample, the overall intake of carbohydrates as a proportion of energy was lower, intake of saturated fatty acids as a proportion of energy was higher, and intake of fiber as both grams and energyadjusted was lower than the current nutrition recommendations (Nordic Nutrition Recommendations 2012: Integrating nutrition and physical activity, 2014) which is common in Finnish adults (Helldán et al., 2013).

It is also important to take into consideration that approximately one third of people eat less than usual during stress (Epel et al., 2004; Oliver \& Wardle, 1999). It remains unclear how many participants in this study population reacted to stress by decreasing eating and to what extent. For example, $73 \%$ reported that when stressed they ate without experiencing physical hunger and this was more common among participants with the highest perceived stress. But, inconsistently, the most stressed persons did not consume more energy than less stressed.

Although several earlier studies have found association between stress and unfavorable diet quality or food choices (Barrington et al., 2014; Groesz et al., 2012; Isasi et al., 2015; Oliver \& Wardle, 
1999; Wardle, Steptoe, Oliver, \& Lipsey, 2000), also opposite findings, in line with our results, have been reported. Perceived stress has not been associated with diet quality measured by indices such as the Alternate Healthy Eating Index (AHEI), the Dietary Approaches to Stopping Hypertension (DASH), the Mediterranean Diet Score (Ferranti et al., 2013), and the Healthy Eating Index-2010 (HEI-2010) (Richardson et al., 2015), or dietary energy density (Grossniklaus et al., 2010), intake of energy from added sugars (Barrington et al., 2014), or omega-3 polyunsaturated fatty acids intake (Suominen-Taipale et al., 2010). Contradictory results may be due to sample differences in gender, ethnic background, or socioeconomic status, for example, and different ways to measure stress and food consumption and nutrient intake as there is no standard method for either of them.

It is also important to take into consideration that the timeframe of measured perceived stress and food consumption and nutrient intake may not fully correspond with each other. Perceived Stress Scale does not measure chronic stress but contains all the previous events that still influence appraised stress (Cohen et al., 1983). Questions in Perceived Stress Scale and Index of Diet Quality concerned the previous month, and in AUDIT-C the previous six months, whereas 48-h dietary recall represents food consumption during a shorter period of time. Nevertheless, we wanted to use 48$\mathrm{h}$ dietary recall to collect food consumption data in as free-living situation as possible so that the measurement would not influence the study participants' dietary intake. Perceived Stress Scale was used because we were interested in participants' subjective experience of stress over a longer period of time.

In this study population, those in the highest perceived stress tertile were less aiming to lose weight and had more depressive symptoms but there were no differences in gender, age, BMI, waist circumference, marital status, number of underage children in household, education level, having regular medication, or type of working hours between the different levels of perceived stress. Also Grossniklaus et al. (Grossniklaus et al., 2010) have found correlation between perceived stress and depressive symptoms in overweight working-age people.

\subsection{Strengths and limitations}

This study was a cross-sectional analysis of the baseline measurements of psychologically distressed overweight and obese persons attending a lifestyle intervention. Despite the fact that all the subjects were, due to the inclusion criteria, psychologically distressed, they still had large variation in Perceived Stress Scale (PSS) scores, which enabled us to investigate the association between the level of stress and features of eating behavior and diet. Using a subjective measure of stress may be debated but in the same Elixir study population higher perceived stress was found to associate with higher objective heart-rate-variability-measured stress on workdays (Föhr et al., 2015). Rather a large sample size and multicenter design, representing three areas in Finland, is also a strength of this study. Furthermore, the study population consisted of ethnically homogenous Finnish population. It is important to study this topic also among populations representing different cultures and ethnic backgrounds to find out whether the findings are culture-specific or modified by ethnic background.

There were also limitations in our study. Participants filled in the electronic questionnaires on their own. This could have encouraged them to answer more truthfully, but it has possibly also increased the misunderstanding of the questions as the responses were not checked with the study personnel, except for the Index of Diet Quality. It is also important to notice that the study population may have been biased, as participants were rather highly educated and most of the participants were non-smokers, married or cohabiting, and aiming to lose weight. Furthermore, the gender distribution did not correspond the typical distribution of overweight and obesity in Finnish population, as overweight is more prevalent in males than females and the prevalence of obesity is equal in both genders (Männistö et al., 2015). Participants were also overall interested in lifestyle changes as they all had enrolled in Elixir lifestyle intervention study voluntarily. These all may affect the generalization of the study results.

\section{Conclusions}

This study provided unique information about the associations of both favorable and unfavorable features of eating behavior and diet quality with perceived stress in psychologically distressed overweight and obese Finns in a non-experimental, real-world context. As hypothesized, higher level of perceived stress was associated with several unfavorable features of eating behavior, such as eating less according to hunger and satiety cues and more according to emotions, but unexpectedly mainly not with the quality or content of the diet measured by 48-h dietary recall of mostly weekdays, Index of Diet Quality, and AUDIT-C. This indicates that either the methods used were incapable to detect stressrelated eating or that this behavior may be compensated during the weekdays. More research and methods development is needed concerning stress and eating.

Highly stressed overweight and obese people were less aiming to lose weight and reported more and severe depressive symptoms. Thus concentrating on psychological aspects and means for handling stress should precede or be included in the nutritional lifestyle interventions and clinical care for this kind of population. Higher prevalence of unfavorable eating behavior in highly stressed overweight and obese people may indicate higher risk for lower quality of diet and unhealthy food choices in addition to difficulties in weight management and eventually higher risk for metabolic health disturbances. More longitudinal research and randomized controlled lifestyle interventions in this area are needed in order to develop efficient methods for the clinicians to assist the overweight and obese patients to handle stress and improve unfavorable eating behavior.

\section{Competing interests}

The authors declare that they have no competing interests.

\section{Acknowledgements}

The Elixir study was supported by the SalWe Research Program for Mind and Body (Tekes - the Finnish Funding Agency for Technology and Innovation grant number 1104/10). The preparation of this paper was also supported by a personal grant (EJ-R) of The Diabetes Research Foundation, and by the PhD student position (EJ$\mathrm{R}$ ) in the Doctoral Programme in Nutrition of the Faculty of Health Sciences, University of Eastern Finland. The funding sources did not have a role in the study design, data collection, data analysis, interpretation of the findings, writing of the article, or the decision to submit the paper for publication.

We would like to thank Sara Mutikainen and Urho Kujala (Department of Health Sciences, University of Jyväskylä) and Elina Mattila (VTT Technical Research Centre of Finland, Tampere, Finland) who have contributed to the Elixir study design and data collection; Eeva Lajunen, Erja Kinnunen, and Maritta Putkiranta for skillful assistance in managing the study visits; Iina Grönberg, Saara Leino, Siniriikka Ventelä, and Raisa Harjula for skillful participation in the conducting the practical period and handling dietary data; Sini Lähteenmäki for data handling and revision, as well as 
Marianna Koivisto, Heidi Aaltonen and Saara Peuhkuri for handling dietary data. We would also like to thank Salla Muuraiskangas for managing the electronic questionnaires of the Elixir study and Anni Helldán (The National Institute for Health and Welfare, Helsinki, Finland) for giving advice for the 48-h dietary recall protocol development.

\section{References}

Aalto, M., Alho, H., Halme, J. T., \& Seppä, K. (2009). AUDIT and its abbreviated versions in detecting heavy and binge drinking in a general population survey. Drug and Alcohol Dependence, 103(1-2), 25-29. http://dx.doi.org/10.1016/ j.drugalcdep.2009.02.013.

Barrington, W. E., Beresford, S. A., McGregor, B. A., \& White, E. (2014). Perceived stress and eating behaviors by sex, obesity status, and stress vulnerability: findings from the vitamins and lifestyle (VITAL) study. Journal of the Academy of Nutrition and Dietetics, 114(11), 1791-1799. http://dx.doi.org/10.1016/ j.jand.2014.03.015.

Beck, A. T., Steer, R. A., Ball, R., \& Ranieri, W. (1996). Comparison of Beck Depression Inventories-IA and -II in psychiatric outpatients. Journal of Personality Assessment, 67(3), 588-597. http://dx.doi.org/10.1207/s15327752jpa6703_13.

Bush, K., Kivlahan, D. R., McDonell, M. B., Fihn, S. D., \& Bradley, K. A. (1998). The AUDIT alcohol consumption questions (AUDIT-C): an effective brief screening test for problem drinking. Ambulatory care quality improvement project (ACQUIP). Alcohol use disorders identification test. Archives of Internal Medicine, 158(16), 1789-1795.

Byrne, S. M. (2002). Psychological aspects of weight maintenance and relapse in obesity. Journal of Psychosomatic Research, 53(5), 1029-1036. pii: S0022399902004877.

Cohen, S. (2015). Dr. Cohen's scales. Retrieved from http://www.psy.cmu.edu scohen/scales.html Accessed 30.07.15.

Cohen, S., Janicki-Deverts, D., \& Miller, G. E. (2007). Psychological stress and disease JAMA, 298(14), 1685-1687. pii: 298/14/1685.

Cohen, S., Kamarck, T. \& Mermelstein, R. (1983). A global measure of perceived stress. Journal of Health and Social Behavior, 24(4), 385-396.

Cohen, S., Kessler, R., \& Gordon, U. (1997). Strategies for measuring stress in studies of psychiatric and physical disorders. In S. Cohen, R. Kessler, \& U. Gordon (Eds.) Measuring stress: A guide for health and social scientists (pp. 3-26). USA: Oxford University Press.

Conner, M., Fitter, M., \& Fletcher, W. (1999). Stress and snacking: a diary study of daily hassles and between-meal snacking. Psychology \& Health, 14(1), 51-63. http://dx.doi.org/10.1080/08870449908407313.

Epel, E., Jimenez, S., Brownell, K., Stroud, L., Stoney, C., \& Niaura, R. (2004). Are stress eaters at risk for the metabolic syndrome? Annals of the New York Academy of Sciences, 1032, 208-210. pii: 1032/1/208.

Ferranti, E. P., Dunbar, S. B., Higgins, M., Dai, J., Ziegler, T. R. Frediani, J. K., ... Brigham, K. L. (2013). Psychosocial factors associated with die quality in a working adult population. Research in Nursing \& Health, 36(3), 242-256. http://dx.doi.org/10.1002/nur.21532.

Föhr, T., Tolvanen, A., Myllymäki, T., Järvelä-Reijonen, E., Rantala, S., Korpela, R.,... Kujala, U. M. (2015). Subjective stress, objective heart rate variability-based stress, and recovery on workdays among overweight and psychologically distressed individuals: a cross-sectional study. Journal of Occupational Medicine and Toxicology (London, England), 10. http://dx.doi.org 10.1186/s12995-015-0081-6, 39-015-0081-6. eCollection 2015.

Goldberg, D. (1972). The detection of psychiatric illness by questionnaire. Maudsley monograph no. 21. Oxford: Oxford University Press.

Groesz, L. M., McCoy, S., Carl, J., Saslow, L., Stewart, J., Adler, N.,... Epel, E. (2012) What is eating you? Stress and the drive to eat. Appetite, 58(2), 717-721. http:/ dx.doi.org/10.1016/j.appet.2011.11.028.

Grossniklaus, D. A., Dunbar, S. B., Tohill, B. C., Gary, R., Higgins, M. K., \& Frediani, J. (2010). Psychological factors are important correlates of dietary pattern in overweight adults. The Journal of Cardiovascular Nursing, 25(6), 450-460. http:// dx.doi.org/10.1097/JCN.0b013e3181d25433.

Heitmann, B. L., \& Lissner, L. (1995). Dietary underreporting by obese individuals-is it specific or non-specific? BMJ (Clinical Research Edition), 311(7011), 986-989.

Helldán, A., Raulio, S., Kosola, M., Tapanainen, H., Ovaskainen, M., \& Virtanen, S. (2013). Finravinto 2012 -tutkimus - The national FINDIET 2012 survey (THL Report No. 16).

Herbert, B. M., Blechert, J., Hautzinger, M., Matthias, E., \& Herbert, C. (2013). Intuitive eating is associated with interoceptive sensitivity. Effects on body mass index. Appetite, 70, 22-30. http://dx.doi.org/10.1016/j.appet.2013.06.082.

Holi, M. M., Marttunen, M., \& Aalberg, V. (2003). Comparison of the GHQ-36, the GHQ-12 and the SCL-90 as psychiatric screening instruments in the Finnish population. Nordic Journal of Psychiatry, 57(3), 233-238. http://dx.doi.org $10.1080 / 08039480310001418$.

Huh, J., Shiyko, M., Keller, S., Dunton, G., \& Schembre, S. M. (2015). The time-varying association between perceived stress and hunger within and between days. Appetite, 89, 145-151. http://dx.doi.org/10.1016/j.appet.2015.02.001.

Isasi, C. R., Parrinello, C. M., Jung, M. M., Carnethon, M. R., Birnbaum-Weitzman, O., Espinoza, R. A.,... Gallo, L. C. (2015). Psychosocial stress is associated with obesity and diet quality in Hispanic/Latino adults. Annals of Epidemiology, 25(2),
84-89. http://dx.doi.org/10.1016/j.annepidem.2014.11.002.

Jääskeläinen, A., Nevanperä, N., Remes, J., Rahkonen, F., Järvelin, M. R., \& Laitinen, J. (2014). Stress-related eating, obesity and associated behavioural traits in adolescents: a prospective population-based cohort study. BMC Public Health, 14. http://dx.doi.org/10.1186/1471-2458-14-321, 321-2458-14-321.

Karlsson, J., Persson, L. O. Sjöström, L. \& Sullivan, M. (2000). Psychometric properties and factor structure of the three-factor eating questionnaire (TFEQ) in obese men and women. Results from the Swedish obese subjects (SOS) study. International Journal of Obesity and Related Metabolic Disorders: Journal of the International Association for the Study of Obesity, 24(12), 1715-1725.

Keskitalo, K., Tuorila, H., Spector, T. D., Cherkas, L. F., Knaapila, A., Kaprio, J., ... Perola, M. (2008). The three-factor eating questionnaire, body mass index, and responses to sweet and salty fatty foods: a twin study of genetic and environmental associations. The American Journal of Clinical Nutrition, 88(2), 263-271. pii: 88/2/263.

Konttinen, H., Männistö, S., Sarlio-Lähteenkorva, S., Silventoinen, K., \& Haukkala, A (2010). Emotional eating, depressive symptoms and self-reported food consumption. A population-based study. Appetite, 54(3), 473-479. http:// dx.doi.org/10.1016/j.appet.2010.01.014.

Krall, J. S., \& Lohse, B. (2010). Cognitive testing with female nutrition and education assistance program participants informs validity of the Satter Eating Competence Inventory. Journal of Nutrition Education and Behavior, 42(4), 277-283. http://dx.doi.org/10.1016/j.jneb.2009.08.003.

Krall, J. S., \& Lohse, B. (2011). Validation of a measure of the Satter Eating Competence Model with low-income females. The International Journal of Behavioral Nutrition and Physical Activity, 8. http://dx.doi.org/10.1186/1479-5868-8-26, 26-5868-8-26.

Laitinen, J., Ek, E., \& Sovio, U. (2002). Stress-related eating and drinking behavior and body mass index and predictors of this behavior. Preventive Medicine, 34(1), 29-39. http://dx.doi.org/10.1006/pmed.2001.0948.

Lappalainen, R., Sairanen, E., Järvelä, E., Rantala, S., Korpela, R. Puttonen, S., ... Kolehmainen, M. (2014). The effectiveness and applicability of different lifestyle interventions for enhancing wellbeing: the study design for a randomized controlled trial for persons with metabolic syndrome risk factors and psychological distress. BMC Public Health, 14. http://dx.doi.org/10.1186/ 1471-2458-14-310, 310-2458-14-310.

de Lauzon, B., Romon, M., Deschamps, V., Lafay, L., Borys, J. M., Karlsson, J.,... Fleurbaix Laventie Ville Sante Study Group. (2004). The threefactor eating questionnaire-R18 is able to distinguish among different eating patterns in a general population. The Journal of Nutrition, 134(9), 2372-2380. pii: $134 / 9 / 2372$

Lee, E. H. (2012). Review of the psychometric evidence of the perceived stress scale. Asian Nursing Research, 6(4), 121-127. http://dx.doi.org/10.1016/ j.anr.2012.08.004.

Leppälä, J., Lagström, H., Kaljonen, A., \& Laitinen, K. (2010). Construction and evaluation of a self-contained index for assessment of diet quality. Scandinavian Journal of Public Health, 38(8), 794-802. http://dx.doi.org/10.1177/ 1403494810382476.

Lohse, B. (2015). The Satter Eating Competence Inventory for low-income persons is a valid measure of eating competence for persons of higher socioeconomic position. Appetite, 87, 223-228. http://dx.doi.org/10.1016/j.appet.2014.12.228.

Lohse, B., Bailey, R. L., Krall, J. S., Wall, D. E., \& Mitchell, D. C. (2012). Diet quality is related to eating competence in cross-sectional sample of low-income females surveyed in Pennsylvania. Appetite, 58(2), 645-650. http://dx.doi.org/10.1016/ j.appet.2011.11.022.

Lohse, B., Satter, E., Horacek, T., Gebreselassie, T., \& Oakland, M. J. (2007). Measuring eating competence: psychometric properties and validity of the ecSatter Inventory. Journal of Nutrition Education and Behavior, 39(5 Suppl.), S154-S166. pii: S1499-4046(07)00690-2.

Männistö, S., Laatikainen, T., Harald, K., Borodulin, K., Jousilahti, P., Kanerva, N., ... Vartiainen, E. (2015). Työikäisten ylipainon ja lihavuuden kasvu näyttää hidastuneen : Kansallisen FINRISKI-terveystutkimuksen tuloksia (In Finnish with an English abstract. The trend towards increasing obesity seems to have slowed down in the working aged Finnish population - results from the National FINRISK Studies). Suomen Lääkärilehti. Finnish Medical Journal, 70(14-15), 969-975.

Nevanperä, N. J., Hopsu, L., Kuosma, E., Ukkola, O., Uitti, J., \& Laitinen, J. H. (2012). Occupational burnout, eating behavior, and weight among working women. The American Journal of Clinical Nutrition, 95(4), 934-943. http://dx.doi.org/ 10.3945/ajcn.111.014191.

Nordic Nutrition Recommendations 2012: Integrating nutrition and physical activity (5th ed.). (2014). Copenhagen: Nordic Council of Ministers.

Ocké, M. C., Larranaga, N., Grioni, S., van den Berg, S. W., Ferrari, P., Salvini, S., ... Slimani, N. (2009). Energy intake and sources of energy intake in the European prospective investigation into cancer and nutrition. European Journal of Clinical Nutrition, 63(Suppl. 4), S3-S15. http://dx.doi.org/10.1038/ ejcn.2009.72.

Oliver, G., \& Wardle, J. (1999). Perceived effects of stress on food choice. Physiology \& Behavior, 66(3), 511-515. doi: S0031-9384(98)00322-9 [pii].

Paturi, M., Nieminen, R., Reinivuo, H., \& Ovaskainen, M. (2006). Ruokien annoskuvakirja. Publications of the National Public Health Institute No. B11.

Psota, T. L., Lohse, B., \& West, S. G. (2007). Associations between eating competence and cardiovascular disease biomarkers. Journal of Nutrition Education and Behavior, 39(5 Suppl.), S171-S178. S1499-4046(07)00756-7.

Racette, S. B., Weiss, E. P., Schechtman, K. B., Steger-May, K., Villareal, D. T., 
Obert, K. A., et al. (2008). Influence of weekend lifestyle patterns on body weight. Obesity (Silver Spring, Md.), 16(8), 1826-1830. http://dx.doi.org/10.1038/ oby.2008.320.

Räikkönen, K., Matthews, K. A., \& Kuller, L. H. (2007). Depressive symptoms and stressful life events predict metabolic syndrome among middle-aged women: a comparison of world health organization, adult treatment panel III, and international diabetes foundation definitions. Diabetes Care, 30(4), 872-877. pii: 30/ $4 / 872$.

Richardson, A. S., Arsenault, J. E., Cates, S. C., \& Muth, M. K. (2015). Perceived stress, unhealthy eating behaviors, and severe obesity in low-income women. Nutrition Journal, 14(1). http://dx.doi.org/10.1186/s12937-015-0110-4, 122-015-01104.

Roininen, K., Lähteenmäki, L., \& Tuorila, H. (1999). Quantification of consumer attitudes to health and hedonic characteristics of foods. Appetite, 33(1), 71-88. http://dx.doi.org/10.1006/appe.1999.0232.

Satter, E. (2007). Eating competence: definition and evidence for the Satter Eating Competence Model. Journal of Nutrition Education and Behavior, 39(5 Suppl.), S142-S153. S1499-4046(07)00094-2.

Stewart-Knox, B. (2014). Eating and stress at work: the need for public health promotion intervention and an opportunity for food product development? Trends in Food Science \& Technology, 35(1), 52-60. http://dx.doi.org/10.1016/j. tifs.2013.10.010.
Suominen-Taipale, A. L., Turunen, A. W., Partonen, T., Kaprio, J., Männistö, S. Montonen, J., ... Verkasalo, P. K. (2010). Fish consumption and polyunsaturated fatty acids in relation to psychological distress. International Journal of Epidemiology, 39(2), 494-503. http://dx.doi.org/10.1093/ije/dyp386.

Torres, S. J., \& Nowson, C. A. (2007). Relationship between stress, eating behavior and obesity. Nutrition (Burbank, Los Angeles County, Calif.), 23(11-12), 887-894. S0899-9007(07)00249-3.

Tylka, T. (2006). Development and psychometric evaluation of a measure of intuitive eating. Journal of Counseling Psychology, 53(2), 226-240. http://dx.doi.org/ 10.1037/0022-0167.53.2.226.

Van Dyke, N., \& Drinkwater, E. J. (2014). Relationships between intuitive eating and health indicators: literature review. Public Health Nutrition, 17(8), 1757-1766. http://dx.doi.org/10.1017/S1368980013002139.

Wallis, D. J., \& Hetherington, M. M. (2009). Emotions and eating. self-reported and experimentally induced changes in food intake under stress. Appetite, 52(2), 355-362. http://dx.doi.org/10.1016/j.appet.2008.11.007.

Wardle, J., Chida, Y., Gibson, E. L., Whitaker, K. L., \& Steptoe, A. (2011). Stress and adiposity: a meta-analysis of longitudinal studies. Obesity (Silver Spring, Md.) 19(4), 771-778. http://dx.doi.org/10.1038/oby.2010.241.

Wardle, J., Steptoe, A., Oliver, G., \& Lipsey, Z. (2000). Stress, dietary restraint and food intake. Journal of Psychosomatic Research, 48(2), 195-202. S0022-3999(00) 00076-3. 